\title{
Stability Analysis of Static Signatures for Automatic Signature Verification
}

\author{
Donato Impedovo and Giuseppe Pirlo \\ Dipartimento di Informatica, Università degli Studi di Bari "A. Moro", via Orabona 4, \\ 70125 - Bari, Italy \\ Centro Interfacoltà "Rete Puglia“, Università degli Studi di Bari "A. Moro", \\ via Giulio Petroni 15F/1, 70100 - Bari, Italy \\ impedovo@deemail.poliba.it
}

\begin{abstract}
This paper presents a static signature verification system based on the concept of local stability. Stable regions are detected in the signatures, during the enrolling phase, and are considered to be those regions affected by low variations of features among the training set. The stability evaluation is based on the Hamming distance. Stable regions are successively used for verification, in the running phase. A region-oriented verification strategy is considered, based on a well-defined similarity measure which takes into account the variability in signing of the writer. The experimental results, carried out on signatures from the GPDS database, demonstrate the viability of proposed approach.
\end{abstract}

Keywords: Biometry, Static Signature Verification, Stability Analysis.

\section{Introduction}

Handwritten signature is a biometric trait with peculiar and interesting characteristics for verification aims. In fact, even if handwritten signature is considered to be a behavioral biometric trait, it depends from physical and psychological conditions of the writer, as well as on the writing device. It follows that automatic signature verification involves aspects from disciplines ranging from human anatomy to engineering, from neuroscience to computer science. Notwithstanding, handwritten signature is wellaccepted by users and well-recognized by public and private institutions. Thus, the field of automatic signature verification is actually attracting more and more researchers, interested to both scientific and commercial aspects [1,2,3].

Two categories of signature verification systems can be considered, depending on the data acquisition method [1]: static (off-line) systems and dynamic (on-line) systems. Static systems perform data acquisition after the writing process has been completed. In this case, the signature is represented by a grey level image $\{\mathrm{I}(\mathrm{u}, \mathrm{v})\}_{0 \leq \mathrm{u} \leq \mathrm{U}, 0 \leq \mathrm{v} \leq \mathrm{v}}$, where $\mathrm{I}(\mathrm{u}, \mathrm{v})$ denotes the grey level at the position $(\mathrm{u}, \mathrm{v})$ of the image. Static systems involve the treatment of the spatio-luminance representation of a signature image. Dynamic systems perform data acquisition during the writing process. In this case on-line acquisition devices are adopted and a signature is 
represented as a sequence $\{\mathrm{S}(\mathrm{n})\}_{\mathrm{n}=0,1 \ldots \mathrm{N}}$, where $\mathrm{S}(\mathrm{n})$ is the signal value sampled at time $\mathrm{n} \cdot \Delta \mathrm{t}$ of the signing process $(0 \leq \mathrm{n} \leq \mathrm{N}), \Delta \mathrm{t}$ being the sampling period. Thus, dynamic systems concern the treatment of a spatio-temporal representation of the signature [3]. Therefore, no direct dynamic information is available on the signing process when static signatures are considered $[4,5]$. Notwithstanding, static signature verification is very important for many application fields, like automatic bank-check processing, insurance form processing, document validation and so on $[6,7,8]$.

In order to improve signature verification performance, this paper presents an attempt to extract useful information derived from the signing process, that can be used for signature verification purposes. The main consideration is that diverse regions of a signature convey different amounts of distinctive information. In particular, stable regions can be considered to better represent the user and at the same time, moreover since their discovering is hidden in the training phase of the system which performs the evaluation among a set of training genuine samples, these regions could be more difficult to imitate than others and imperfections of the forgers could be much more easily detectable. Thus signature verification can be performed more effectively if stable regions are considered $[9,10,11,12]$.

Starting from this consideration, in this paper a technique for the analysis of local stability is used to detect stable regions in a static signature. A signature verification system is then presented which performs signature verification using only stable regions for verification. The organization of the paper is the following. Section 2 presents the stability analysis technique. Section 3 presents the static signature verification system. Section 4 reports the experimental results, obtained on the GPDS database. The conclusion is reported in Section 5.

\section{Analysis of Stability in Static Signatures}

It is common experience that not all parts of a signature are equally distinctive for signature verification, as widely discussed in recent literature [13, 14, 15]. In order to select distinctive parts of a static signature, in this paper regions on the upper and lower contours of signature are considered (at a first stage), since it is well known that upper and lower contours of a signature can convey relevant information for verification aims. Therefore, after the preprocessing phase, in which each signature is binarized and normalized to a fixed rectangular area, stable regions are detected. More precisely, let $I=I(u, v)$ be a signature image, $0 \leq u \leq U^{\max }, 0 \leq v \leq V^{\max } ; I_{x, y}=$ $I(x+r, y+s)$ a sub-image of size $(2 R+1,2 S+1)(R, S$ integers $)$ with $-R \leq r \leq R,-S \leq s \leq$ $S$. Furthermore, let $I^{I}, I^{2}, \ldots, I^{k}, \ldots I^{K}$ be a set of $K$ genuine signature images and $I_{x_{p}^{k}, y_{p}^{k}}^{k}$ be the $p$-th region extracted from $I^{k}$. The stability $S\left(I_{x_{p}^{k}, y_{p}^{k}}^{k}\right)$ of the region $I_{x_{p}^{k}, y_{p}^{k}}^{k}$ is defined as the mean value of the distance between $I_{x_{p}^{k}, y_{p}^{k}}^{k}$ and the corresponding regions on the other genuine signatures [16]:

$$
S\left(I_{x_{p}^{k}, y_{p}^{k}}^{k}\right)=\operatorname{mean}\left\{D^{*}\left(I_{x_{p}^{k}, y_{p}^{k}}^{k}, I_{x_{p}^{t}, y_{p}^{t}}^{t}\right) \mid t=1,2, \ldots, K, k \neq t\right\}
$$




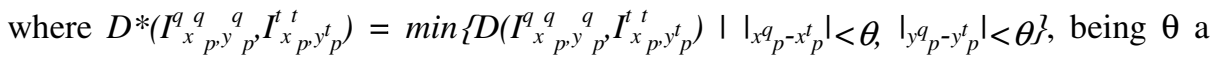
threshold and $\mathrm{D}(\cdot)$ the Hamming Distance among the sub-images. Figure 1 shows the matching procedure, in which the $p$-th sub-image of the $k$-th signature is matched against the corresponding sub-image of the $t$-th signature, determined within a wide region. Of course, this procedure allows to assign to each sub-region a degree of stability. Regions that are perfectly replicated by the signer on different genuine signatures will have a stability value (distance value) equal to zero. Conversely, as the sub-region matches worse with the corresponding sub-regions, the distance augments. This means that the signer is not stable in affixing that part of the signature. The stability parameter $S(\cdot)$ ranges in the interval $[0,1]$ and the boundary 0 and 1 represents, respectively, the highest and the lowest degree of similarity.

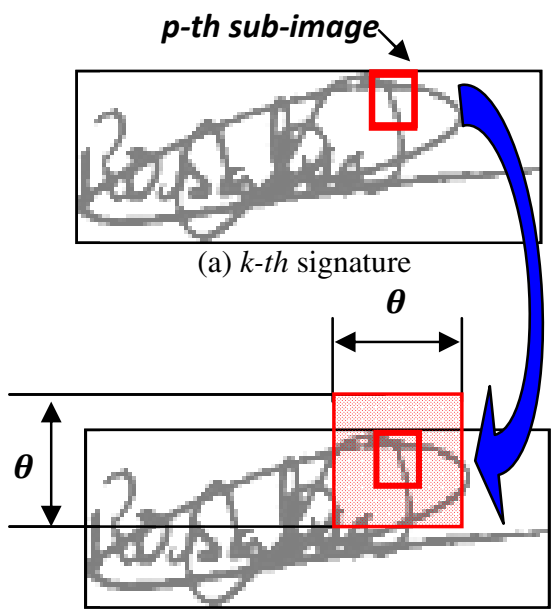

(b) $t$-th signature

Fig. 1. Example of Matching Procedure

\section{Stability-Based Signature Verification}

The signature verification system consists of four main modules. The Data Acquisition Module (DAM) allows data acquisition of static signatures. The Preprocessing Module (PM) performs signature binarization, signature normalization to a fixed rectangular area and noise reduction. The Feature Extraction Module (FEM) performs the extraction of the discriminative features. More precisely, in the enrollment phase the stable regions of the signature are extracted, according to the approach described in Section 2. The Classification Module (CM) performs signature verification according to a two-level strategy [15]: first it applies a matching rule on stable regions, based on a simple similarity measure, to derive verification responses at the region-level; successively, local decisions are combined to obtain the final decision at the signature level. In the first stage the stable regions on the unknown signature are individually verified. For this purpose, each region is matched against the corresponding regions on the genuine samples. In the regional matching stage a 
set of $\mathrm{N}$ sub-images are extracted from the test signature image and compared against $\mathrm{N}$ corresponding stable sub-images on the reference signature images. Let $I^{t}$ be the image of a test signature and $I^{I}, I^{2}, \ldots, I^{k}, \ldots I^{K}$ be a set of $K$ reference signature images.

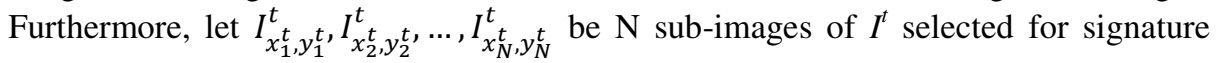
matching and corresponding to stable regions detected in the training phase. A subimage $I_{x_{n}{ }_{n}, y_{n}{ }_{n}}$ of the test signature is considered as belonging to a genuine signature if and only if

$$
\left|\Delta\left(I_{x_{n}^{t}, y_{n}^{t}}^{t}\right)-\mu_{n}\right| \leq \sigma_{n}
$$

where:

$$
\Delta\left(I_{x_{n}^{t}, y_{n}^{t}}^{t}\right)=\min \min D\left(I_{x_{n}^{t}, y_{n}^{t}}^{t}, I_{x_{n}^{k}, y_{n}^{k}}^{k}\right)
$$

with $\left.\right|_{x^{k}}{ }^{-x^{v}}{ }_{p} \mid<\theta$ and $\left|y_{p^{k}}{ }^{-y^{v}}{ }_{p}\right|<\theta, \mu_{\mathrm{n}}$ and $\sigma_{\mathrm{n}}$ are the mean and the standard deviation

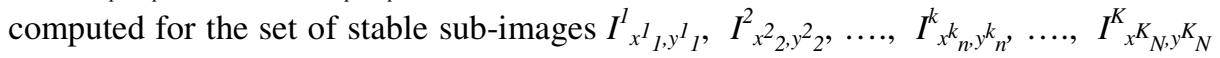
of the genuine signatures.

In the second stage a majority voting strategy is used to combine local decisions and produce the final verification decision, the signature is considered to be genuine if and only if $R_{g} \geq \lambda_{s} \cdot R_{\text {tot }}$, where $\mathrm{R}_{\mathrm{g}}$ is the number of genuine sub-regions, $\mathrm{R}_{\mathrm{tot}}$ is the total number of sub-regions considered for signature verification and $\lambda_{\mathrm{s}}$ is a personal threshold value of the signer which estimates his/her personal variability in signing.

\section{Experimental Results}

The GPDS database has been considered: 16200 signatures from 300 individuals have been used. In particular, for each individual, there are 24 genuine signatures and 30 forgeries [17]. Each signature was normalized to a box of $113 \times 43$ pixels, the subregions inspected in order to evaluate their stability have a size of $11 \times 11$ pixels. For each signer the most stable regions are identified according to the procedure described in Section 2, and at a first stage, the number of stable regions selected by the described approach range from 3 to 6 for each signature.

Results are reported in table 1 in terms of Type I error rate (i.e. False Rejection Rate - FRR) and of Type II error rate (i.e. False Acceptance Rate - FAR). Moreover FAR is evaluated both considering skilled and random forgeries. The first, second and third rows refer, respectively, to the use of regions selected on the lower, upper and lower.and.upper contour of the signature.

Table 1. Error rate

\begin{tabular}{cccc}
\hline & FRR & FAR-skilled & FAR-random \\
\hline Lower Contour & $40 \%$ & $31 \%$ & $17 \%$ \\
Upper Contour & $22 \%$ & $25 \%$ & $13 \%$ \\
Lower+Upper Contour & $25 \%$ & $26 \%$ & $22 \%$ \\
\hline
\end{tabular}


One of the biggest problem, due to the use of the upper and lower contours in order to investigate stable regions, is related to the simplicity and linearity of the contour. In fact if, for instance, the lower contour is an underlining trait, each region on that contour will result in a very high stability (low distance), but at the same time it will be not discriminative. To partially overcame this problem, the stability of each portion of the signature could be evaluated. Figure 2 shows the stability of regions of two static signatures: high stability refers to regions showing little distance values. It can be observed that circular and straight traits have an high degree of stability.
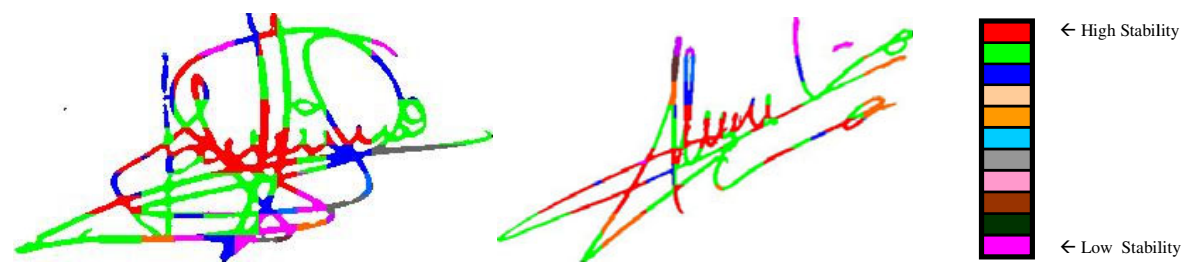

Fig. 2. The stability is evaluated by taking into account each portion of the signature

However, since it is well known that, in general, the upper and the lower contour can provide useful features for signature matching and in order to reduce the computational load due to the inspection of each portion of the signature, the inspection of regions located in middle part of the signature is performed when the upper (lower) part of the profile containing the stable regions is evaluated to be a linear or a circular one. To this aim, the trajectory of the upper (lower) profile has been evaluated by considering the derivatives of traits on consecutive windows. According to this procedure, if the region is considered to convey not discriminative features, the middle part of the signature is inspected (figure 3).

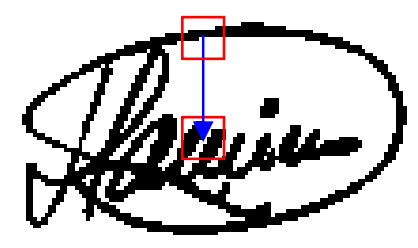

Fig. 3. Skipping not discriminative traits

Under these assumptions the Type I error rate is $20 \%$, whereas the type II error rates for random and skilled forgeries is respectively $19 \%$ and $22 \%$. These results are comparable with other results on the same database carried out in the recent literature [18].

It must be underlined that a user-dependent threshold can be adopted to select specific stable regions starting from $S(\cdot)(1)$. The evaluation of $S(\cdot)$ could be improved by comparing the ability of forgery in replicating the specific region under analysis. This should be taken into account in future works. 


\section{Conclusion}

This paper presents a new technique for the analysis of stability in static signatures, based on Hamming Distance. In particular stable regions are detected by comparing multiple genuine signatures. These regions are then considered for automatic signature verification and a local verification decision is derived for each region. A Majority Vote schema is finally used to combine decisions achieved at regional level. The experimental results, carried out on signatures from the GPDS database, demonstrate that the proposed approach can be considered as a first implementation of a local stability technique. Of course the approach needs more research, in fact the definition of stability must take into account also the discrimination capability of the specific region, to this aim forgeries samples could be considered in the training phase.

\section{References}

1. Plamondon, R., Lorette, G.: Automatic Signature Verification and Writer Identification The State of the Art. Pattern Recognition 22(2), 107-131 (1989)

2. Leclerc, F., Plamondon, R.: Automatic Signature Verification: The State of the Art - 1989 1993. In: Plamondon, R. (ed.) IJPRAI, vol. 8(3), pp. 643-660. World Scientific, Singapore (1994)

3. Impedovo, D., Pirlo, G.: Automatic Signature Verification - The State of the Art. IEEE Transactions on Systems, Man and Cybernetics - Part C: Applications and Review 38(5), 609-635 (2008)

4. Plamondon, R., Srihari, S.N.: On line and Off line Handwriting Recognition: A Comprehensive Survey. IEEE T-PAMI 22(1), 63-84 (2000)

5. Dimauro, G., Impedovo, S., Lucchese, M.G., Modugno, R., Pirlo, G.: Recent Advancements in Automatic Signature Verification. In: 9th International Workshop on Frontiers in Handwriting Recognition (IWFHR-9), Kichijoji, October 25-29, pp. 179-184 (2004)

6. Dimauro, G., Impedovo, S., Pirlo, G., Salzo, A.: A multi-expert signature verification system for bankcheck processing. International Journal of Pattern Recognition and Artificial Intelligence (IJPRAI) 11(5), 827-844 (1997)

7. Yoshimura, M., Yoshimura, I.: Investigation of a verification system for Japanese countersignatures on traveler's checks. Trans. IEICE J80-D-II(7), 1764-1773 (1997)

8. Lee, L.L., Lizarraga, M.G., Gomes, N.R., Koerich, A.L.: A prototype for Brazilian bankcheck recognition. IJPRAI 11(4), 549-569 (1997)

9. Ferrer, M.A., Alonso, J.B., Travieso, C.M.: Offline Geometric Parameters for Automatic Signature Verification Using Fixed-Point Arithmetic. IEEE T-PAMI 27(6), 993-997 (2005)

10. Nouboud, F.: Handwritten signature verification: a global approach. In: Impedovo, S. (ed.) Fundamentals in Handwriting Recognition, pp. 455-459. Springer, Heidelberg (1994)

11. Ramesh, V.E., Narasimha Murty, M.: Off-line signature verification using genetically optimized weighted features. Pattern Recognition 32(2), 217-233 (1999)

12. Bajaj, R., Chaudhury, S.: Signature Verification Using Multiple Neural Classifiers. Pattern Recognition 30(1), 1-7 (1997) 
13. Congedo, G., Dimauro, G., Forte, A.M., Impedovo, S., Pirlo, G.: Selecting Reference Signatures for On Line Signature Verification. In: Braccini, C., Vernazza, G., DeFloriani, L. (eds.) ICIAP 1995. LNCS, vol. 974, pp. 521-526. Springer, Heidelberg (1995)

14. Impedovo, D., Modugno, R., Pirlo, G., Stasolla, E.: Handwritten Signature Verification by Multiple Reference Sets. In: Proc. of the 11th International Conference on Frontiers in Handwriting Recognition (ICFHR), pp. 19-21 (August 2008)

15. Impedovo, D., Pirlo, G.: On the Measurement of Local Stability of Handwriting - An application to Static Signature Verification. In: Proc. of Biometric Measurements and Systems for Security and Medical Applications (BIOMS 2010), Taranto, Italy, pp. 41-44. IEEE Computer Society Press, Los Alamitos (2010)

16. Pirlo, G., Impedovo, D., Stasolla, E., Trullo, C.A.: Learning local correspondences for static signature verification. In: Serra, R., Cucchiara, R. (eds.) AI*IA 2009. LNCS, vol. 5883, pp. 385-394. Springer, Heidelberg (2009)

17. Vargas, J.F., Ferrer, M.A., Travieso, C.M., Alonso, J.B.: Off-line Handwritten Signature GPDS-960 Corpus. In: Proc. 9th ICDAR, September 23-26, vol. 2, pp. 764-768 (2007)

18. Jayadevan, R., Kolhe, S.R., Patil, P.M.: Dynamic Time Warping Based Static Hnad Printed Signature Verification. Journal of Pattern Recognition Research 4(1), 52-65 (2009) 